\title{
In One and Two Dimensions, Every Stationary Measure for a Stochastic Ising Model is a Gibbs State
}

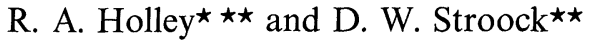 \\ Department of Mathematics, University of Colorado, Boulder, Colorado 80309, USA
}

\begin{abstract}
It is shown that one and two dimensional (generalized) stochastic Ising models with finite range potentials have only Gibbs states as their stationary measures. This is true even if the stationary measure or the potential is not translation invariant. This extends previously known results which are restricted to translation invariant stationary measures and potentials. In particular if the potential has only one Gibbs state the stochastic Ising Model must be ergodic.
\end{abstract}

\section{Introduction}

One of the unpleasant aspects of studying Gibbs states with stochastic Ising models is that one does not know, in general, if uniqueness of phase implies that any associated stochastic Ising model is ergodic. The converse statement, namely that ergodicity of some associated stochastic Ising model implies uniqueness of phase, is well-known and is one of the major reasons for the interest in stochastic Ising models (cf. [5] for example). The purpose of the present paper is to show that in one and two dimensions every stationary measure for any reasonable stochastic Ising model associated with a given potential energy function is a Gibbs state for that potential. In particular, this result resolves the question mentioned above, at least in one and two dimensions.

The technique on which our proof turns is that of considering how the free energy of a state evolves under the action of the stochastic Ising model. This idea has been used before in the study of similar questions (cf. [1], [2], and [7]). In these earlier applications, the free energy functional was used as a kind of Liapounov function. Unfortunately, as a Liapounov function the free energy functional isn't completely satisfactory unless everything, including the initial state, is assumed to be translation invariant. Our application of the free energy functional is quite different. In particular, we will be concerned with the free energy of a state in a finite

* Research supported in part by N.S.F. Grant MPS 74-18926

$\star \star \quad$ Alfred P. Sloan Fellow 
box; and our proof rests on the simple observation that if the state is stationary for the stochastic Ising model, then the free energy of that state in each finite box is constant under the action of the stochastic Ising model. In this connection, we want to mention our indebtedness to the work of Moulin Ollagnier and Pinchon in [7]. It is the elegance of their expression for the free energy of a state in a box which enabled us to carry out our computations.

Finally, we must admit that we cannot explain why dimension seems to play such an important rôle. In fact, we do not even know whether it really does or merely appears to as a consequence of our technique. The only thing of which we are sure is that our argument as it stands cannot be extended to three or more dimensions. Amplification of this point is given in the last section.

\section{The Main Result}

Given $d \geqq 1$, let $Z^{d}$ be the $d$-dimensional square lattice and put $E=E_{d}=(\{-1,1\})^{Z^{d}}$. We think of $E$ as a compact metric space with the obvious product topology; and we use $C(E)$ and $M(E)$ to denote the set of all continuous real-valued functions on $E$ and the set of all probability measures on $E$, respectively. $\hat{E}=\hat{E}_{d}$ stands for the class of all finite subsets of $Z^{d}$, and for each $F \in \hat{E}$ we define $\chi_{F} \in C(E)$ by

$$
\chi_{F}(\eta)=\prod_{k \in F} \eta_{k}
$$

where $\eta_{k}, k \in Z^{d}$, denotes the $k^{\text {th }}$ coordinate of $\eta \in E$. Given $\Lambda \in \hat{E}$ and $A \subseteq \Lambda$, define

$$
[A, \Lambda]=\left\{\eta \in E: \eta_{k}=1 \text { if } k \in A \text { and } \eta_{k}=-1 \quad \text { if } k \in \Lambda \backslash A\right\} \text {. }
$$

Given $L \geqq 1$, we say that $\left\{J_{F}: F \in \hat{E}\right\} \cong R^{1}$ is a potential with range $L$ if

i) for all $k \in Z^{d}$ and all $F \in E^{F}$ such that $k \in F$ and $J_{F} \neq 0$,

$$
\text { ii) } \sup _{k \in Z^{d}} \sum_{F \ni k}\left|J_{F}\right|<\infty \text {. }
$$

(It should be observed that we have not insisted that $\left\{J_{F}: F \in Z^{d}\right\}$ be translation invariant in the sense demanded in [1], [2], and [7].) Given such a potential, we say that $\mu \in M(E)$ is a Gibbs state with potential $\left\{J_{F}: F \in Z^{d}\right\}$ if for all $k \in Z^{d}, \Lambda \in \hat{E}$ with $k \in \Lambda$, and $A \subseteq \Lambda$ :

$$
\int_{[A, \Lambda]} \exp \left[\sum_{F \ni k} \mathrm{~J}_{F} \chi_{F}(\eta)\right] \mu(d \eta)=\int_{\left[A_{k}, \Lambda\right]} \exp \left[\sum_{F \ni k} J_{F} \chi_{F}(\eta)\right] \mu(d \eta)
$$

where

$$
A_{k} \equiv A \Delta\{k\} .
$$

It is easy to check that the preceding definition coincides with the more familiar one in terms of conditional probabilities.

Finally, given a potential $\left\{J_{F}: F \in \hat{E}\right\}$ with range $L$, we will say that the coefficients $\left\{c_{k}: k \in Z^{d}\right\} \subseteq C(E)$ are associated with $\left\{J_{F}: F \in \hat{E}\right\}$ if the functions

$$
b_{k}(\cdot) \equiv c_{k}(\cdot) \exp \left[-\sum_{F \ni k} J_{F} \chi_{F}(\cdot)\right]
$$


are strictly positive and have the properties that:

i) $b_{k}(\eta)=b_{k}\left(\eta^{\prime}\right)$ whenever $\eta_{l}=\eta_{l^{\prime}}$ for all $0<|l-k|<L$,

ii) $0<\inf _{k} \inf _{\eta} b_{k}(\eta) \leqq \sup _{k} \sup _{\eta} b_{k}(\eta)<\infty$.

For $k \in Z^{d}$ and $f \in C(E)$, define $\Delta_{k} f(\eta)=f\left({ }_{k} \eta\right)-f(\eta), \eta \in E$, where

$$
{ }_{k} \eta_{l}=\left\{\begin{array}{rll}
\eta_{l} & \text { if } l \neq k \\
-\eta_{k} & \text { if } l=k
\end{array}\right.
$$

and define $\mathscr{D}=\left\{f \in C(E): \Delta_{k} \mathrm{f} \equiv 0\right.$ for all but a finite number of $\left.k \in Z^{d}\right\}$. We will say that the operator $\mathscr{L}$ defined on $\mathscr{D}$ by

$$
\mathscr{L} f(\cdot)=\sum_{k \in Z^{d}} c_{k}(\cdot) \Delta_{k} f(\cdot)
$$

is associated with the potential $\left\{J_{F}: F \in \hat{E}\right\}$ if the coefficients $\left\{c_{k}: k \in Z^{d}\right\}$ are associated with $\left\{J_{E}: F \in \hat{E}\right\}$. It is easy to check that $\mu \in M(E)$ is a Gibbs state with potential $\left\{J_{F}: F \in E\right\}$ if and only if

(1.4) $\int f \mathscr{L} g d \mu=\int g \mathscr{L} f d \mu$

for all $f, g \in \mathscr{D}$. Moreover, there is a unique Feller semi-group $\left\{T_{t}: t \geqq 0\right\}$ on $C(E)$ with the property that

$$
T_{t} f-f=\int_{0}^{t} T_{s} \mathscr{L} f d s, \quad t \geqq 0 \text { and } f \in \mathscr{D} .
$$

Finally, (1.4) is equivalent to

$$
\int f T_{t} g d \mu=\int g T_{t} f d \mu, \quad t \geqq 0 \text { and } f, g \in C(E) .
$$

Proof of these and related facts can be found in [4] and [6]. If $\left\{T_{t}: t \geqq 0\right\}$ is the unique Feller semi-group satisfying (1.5) for some $\mathscr{L}$ associated with $\left\{J_{F}: F \in \hat{E}\right\}$, we will call it a stochastic Ising model with potential $\left\{J_{F}: F \in \hat{E}\right\}$.

Theorem 1.7. If $d=1$ or $2,\left\{J_{F}: F \in \hat{E}\right\}$ is a potential with range $L$, and $\left\{T_{t}: t \geqq 0\right\}$ is a stochastic Ising model associated with $\left\{J_{F}: F \in \hat{E}\right\}$, then $\mu \in M(E)$ is stationary for $\left\{T_{t}: t \geqq 0\right\}$ (i.e. $T_{t}^{*} \mu=\mu$ for all $t \geqq 0$ ) if and only if $\mu$ is a Gibbs state with potential $\left\{J_{F}: F \in \hat{E}\right\}$.

The "if" assertion is obvious, in any dimension, from (1.6); simply take $g \equiv 1$ and observe that $T_{t} g \equiv 1$ for all $t \geqq 0$.

To prove the "only if" part, we first point out that $\mu$ is a Gibbs state if and only if

$$
\int_{[A, A]} c_{k} d \mu=\int_{\left[A_{k}, A\right]} c_{k} d \mu
$$

for all $k \in Z^{d}, k \in \Lambda \in \hat{E}$, and $A \cong \Lambda$. Next, we introduce the following notation:

$$
\begin{aligned}
\Delta_{n} & =\left\{k \in Z^{d}:|k|<n L\right\}, \quad n \geqq 1, \\
U_{n}(\cdot) & =\sum_{F \subseteq \Lambda_{n}} J_{F} \chi_{F}(\cdot), \quad n \geqq 1, \\
F_{n}(\mu) & =\int U_{n} d \mu+\sum_{A \subseteq \Lambda_{n}} \mu\left(\left[A, \Lambda_{n}\right]\right) \log \mu\left(\left[A, \Lambda_{n}\right]\right), \quad n \geqq 1 \quad \text { and } \mu \in M(E) .
\end{aligned}
$$


The number $F_{n}(\mu)$ is the free energy of $\mu$ in the box $\Lambda_{n}$. Given $\mu \in M(E)$, set $\mu_{t}=T_{t}^{*} \mu$, $t \geqq 0$. The rest of our proof turns on the fact that

(1.9) $\left.\frac{d F_{n}\left(\mu_{t}\right)}{d t}\right|_{t=0}=0, \quad n \geqq 1$,

if $\mu$ is stationary and the expression given in the next lemma for $\left.\frac{d F_{n}\left(\mu_{t}\right)}{d t}\right|_{t=0}$

Lemma 1.10. (Moulin Ollagnier and Pinchon): If $\mu \in M(E)$ has the property that $\mu\left(\left[A, \Lambda_{n}\right]\right)>0$ for all $A \subseteq \Lambda_{n}$, then

$$
\begin{aligned}
& \left.2 \frac{d F_{n}\left(\mu_{t}\right)}{d t}\right|_{t=0} \\
& =-\sum_{k \in \Lambda_{n}} \sum_{A \subseteq A_{n}}\left(\Gamma_{n}(k, A)-\Gamma_{n}\left(k, A_{k}\right)\right) \log \frac{\Gamma_{n}(k, A)}{\Gamma_{n}\left(k, A_{k}\right)} \\
& \quad+\sum_{k \in \Lambda_{n}} \sum_{A \subseteq \Lambda_{n}}\left(\Gamma_{n}(k, A)-\Gamma_{n}\left(k, A_{k}\right)\right)\left(V_{n}(k, A)+\log \frac{\Gamma_{n}(k, A)}{\mu\left(\left[A, \Lambda_{n}\right]\right)}\right. \\
& \left.-\log \frac{\Gamma_{n}\left(k, A_{k}\right)}{\mu\left(\left[A_{k}, \Lambda_{n}\right]\right)}\right)
\end{aligned}
$$

where

and

$$
\Gamma_{n}(k, A)=\int_{\left[A, \Lambda_{n}\right]} c_{k} d \mu
$$

$$
V_{n}(k, A)=-2 \sum_{\substack{F \subseteq A_{n} \\ F \ni k}}(-1)^{\left|F \cap\left(A_{n} \backslash A\right)\right|} J_{F} .
$$

Proof. First note that

$$
\left.\frac{d}{d t} \int U_{n} d \mu_{t}\right|_{t=0}=\int \mathscr{L} U_{n} d \mu=\sum_{k \in \Lambda_{n}} \sum_{A \subseteq A_{n}} U_{n}(A)\left(\Gamma_{n}\left(k, A_{k}\right)-\Gamma_{n}(k, A)\right),
$$

where

$$
U_{n}(A) \equiv \sum_{F \subseteq \Lambda_{n}}(-1)^{\left|F \cap\left(\Lambda_{n} \backslash A\right)\right|} J_{F}
$$

By an easy change of variables, we have:

$$
\sum_{A \subseteq A_{n}} U_{n}(A)\left(\Gamma_{n}\left(k, A_{k}\right)-\Gamma_{n}(k, A)\right)=\sum_{A \subseteq A_{n}}\left(U_{n}\left(A_{k}\right)-U_{n}(A)\right) \Gamma_{n}(k, A) .
$$

But

and so

$$
\begin{aligned}
U_{n}\left(A_{k}\right)-U_{n}(A) & =\sum_{F \subseteq \Lambda_{n}}\left((-1)^{|F \cap\{k\}|}-1\right)(-1)^{\left|F \cap\left(A_{n} \backslash A\right)\right|} J_{F} \\
& =-2 \sum_{\substack{F \subseteq A_{n} \\
F \ni k}}(-1)^{\left|F \cap\left(A_{n} \backslash A\right)\right|} J_{F}=V_{n}(k, A),
\end{aligned}
$$

$$
\left.\frac{d}{d t} \int U_{n} d \mu_{t}\right|_{t=0}=\sum_{k \in \Lambda_{n}} \sum_{A \subseteq A_{n}} V_{n}(k, A) \Gamma_{n}(k, A) .
$$


Next, because $\mu\left(\left[A, \Lambda_{n}\right]\right)>0$ for all $A \subseteq \Lambda_{n}$,

$$
\begin{aligned}
& \left.\frac{d}{d t} \sum_{A \subseteq A_{n}} \mu_{t}\left(\left[A, \Lambda_{n}\right]\right) \log \mu_{t}\left(\left[A, \Lambda_{n}\right]\right)\right|_{t=0} \\
& =\sum_{A \subseteq \Lambda_{n}}\left(1+\log \mu\left(\left[A, \Lambda_{n}\right]\right)\right) \int \mathscr{L} I_{\left[A, \Lambda_{n}\right]} d \mu \\
& =\sum_{k \in \Lambda_{n}} \sum_{A \subseteq A_{n}} \log \mu\left(\left[A, \Lambda_{n}\right]\right)\left(\Gamma_{n}\left(k, A_{k}\right)-\Gamma_{n}(k, A)\right)
\end{aligned}
$$

since

$$
\sum_{A \subseteq A_{n}} \int \mathscr{L} I_{\left[A, A_{n}\right]} d \mu=\int \mathscr{L} 1 d \mu=0 .
$$

Changing variables, we now obtain:

$$
\left.\frac{d}{d t} \sum_{A \subseteq A_{n}} \mu_{t}\left(\left[A, \Lambda_{n}\right]\right) \log \mu_{t}\left(\left[A, \Lambda_{n}\right]\right)\right|_{t=0}=\sum_{k \in \Lambda_{n}}\left(\log \frac{\mu\left(\left[A_{k}, \Lambda_{n}\right]\right)}{\mu\left(\left[A, \Lambda_{n}\right]\right)}\right) \Gamma_{n}(k, A) .
$$

We have therefore proved that

$$
\left.\frac{d}{d t} F_{n}\left(\mu_{t}\right)\right|_{t=0}=\sum_{k \in A_{n}} \sum_{A \subseteq A_{n}}\left(V_{n}(k, A)+\log \frac{\mu\left(\left[A_{k}, \Lambda_{n}\right]\right)}{\mu\left(\left[A, \Lambda_{n}\right]\right)}\right) \Gamma_{n}(k, A) .
$$

Since

$$
V_{n}(k, A)=-V_{n}\left(k, A_{k}\right)
$$

and

$$
\log \frac{\mu\left(\left[A_{k}, \Lambda_{n}\right]\right)}{\mu\left(\left[A, \Lambda_{n}\right]\right)}=-\log \frac{\mu\left(\left[A, \Lambda_{n}\right]\right)}{\mu\left(\left[A_{k}, \Lambda_{n}\right]\right)},
$$

(1.14) can be re-written:

(1.15) $2 \frac{d}{d t} F_{n}\left(\mu_{t}\right)_{t=0}=\sum_{k \in \Lambda_{n}} \sum_{A \subseteq A_{n}}\left(V_{n}(k, A)-\log \frac{\mu\left(\left[A, \Lambda_{n}\right]\right)}{\mu\left(\left[A_{k}, \Lambda_{n}\right]\right)}\right)\left(\Gamma_{n}(k, A)-\Gamma_{n}\left(k, A_{k}\right)\right)$, and (1.11) follows easily from (1.15) after an obvious manipulation.

Lemma 1.16. If $\mu \in M(E)$ is stationary for $\left\{T_{t}: t \geqq 0\right\}$, then $\mu([A, \Lambda])>0$ for all $\Lambda \in \hat{E}$ and $A \subseteq \Lambda$. Moreover, for all $n \geqq 1$,

$$
\begin{aligned}
& \sum_{k \in \Lambda_{n}} \sum_{A \subseteq A_{n}}\left(\Gamma_{n}(k, A)-\Gamma_{n}\left(k, A_{k}\right)\right) \log \frac{\Gamma_{n}(k, A)}{\Gamma_{n}\left(k, A_{k}\right)} \\
& =\sum_{k \in A_{n}} \sum_{A \subseteq A_{n}}\left(\Gamma_{n}(k, A)-\Gamma_{n}\left(k, A_{k}\right)\right)\left(V_{n}(k, A)+\log \frac{\Gamma_{n}(k, A)}{\mu\left(\left[A, A_{n}\right]\right)}\right. \\
& \left.-\log \frac{\Gamma_{n}\left(k, A_{k}\right)}{\mu\left(\left[A_{k}, A_{n}\right]\right)}\right) .
\end{aligned}
$$

In particular, there is a $K<\infty$ such that if $\partial \Lambda_{n}=\Lambda_{n} \backslash \Lambda_{n-1}, n \geqq 2$, then

$$
\begin{aligned}
& \sum_{k \in A_{n}} \sum_{A \subseteq A_{n}}\left(\Gamma_{n}(k, A)-\Gamma_{n}\left(k, A_{k}\right)\right) \log \frac{\Gamma_{n}(k, A)}{\Gamma_{n}\left(k, A_{k}\right)} \\
& \leqq K \sum_{k \in \partial A_{n}} \sum_{A \subseteq A_{n}}\left|\Gamma_{n}(k, A)-\Gamma_{n}\left(k, A_{k}\right)\right| .
\end{aligned}
$$


Proof. Suppose that $\mu$ is stationary and that $\mu([A, \Lambda])=0$ for some $\Lambda$ and $A \subset \Lambda$. Then

$$
0=\frac{d}{d t} \mu_{t}\left(I_{[A, \Lambda]}\right)=\int \mathscr{L} I_{[A, \Lambda]} d \mu=\int \sum_{k \in \Lambda} c_{k}\left[I_{\left[A_{k}, A\right]}-I_{[A, \Lambda]}\right] d \mu .
$$

Since $c_{k}>0$ this implies that $\mu\left(\left[A_{k}, \Lambda\right]\right)=0$ for all $k \in \Lambda$. Repeating this argument we see that for all $B \subset \Lambda, \mu([B, \Lambda])=0$. This is a contradiction and thus $\mu([A, \Lambda])>0$ for all $\Lambda$ and all $A \subset \Lambda$.

Equation (1.17) is an immediate consequence of Lemma 1.10 and the fact that $d F_{n}\left(\mu_{t}\right) / d t \equiv 0$.

Finally, the estimate (1.18) follows from (1.17) once one notices that

$$
\frac{1}{2} V_{n}(k, A)=-\log \frac{\Gamma_{n}(k, A)}{\mu\left(\left[A, \Lambda_{n}\right]\right)}=\log \frac{\Gamma_{n}\left(k, A_{k}\right)}{\mu\left(\left[A_{k}, \Lambda_{n}\right]\right)}
$$

for $k \in \Lambda_{n-1}$ and that

$$
K \equiv \sup _{n \geqq 1} \sup _{k \in \Lambda_{n}} \sup _{A \subseteq \Lambda_{n}}\left|V_{n}(k, A)+\log \frac{\Gamma_{n}(k, A)}{\mu\left(\left[A, \Lambda_{n}\right]\right)}-\log \frac{\Gamma_{n}(k, A)}{\mu\left(\left[A, \Lambda_{n}\right]\right)}\right|<\infty .
$$

Next define for $n \geqq 1$ and $k \in \Lambda_{n}$ :

$$
\alpha_{n}(k)=\sum_{A \subseteq \Lambda_{n}}\left(\Gamma_{n}(k, A)-\Gamma_{n}\left(k, A_{k}\right)\right) \log \frac{\Gamma_{n}(k, A)}{\Gamma_{n}\left(k, A_{k}\right)}
$$

and

$$
\beta_{n}(k)=\sum_{A \subseteq A_{n}}\left|\Gamma_{n}(k, A)-\Gamma_{n}\left(k, A_{k}\right)\right|
$$

As a consequence of Jensen's inequality applied to the function $(x, y) \rightarrow(x-y) \log \frac{x}{y}$ on $(0, \infty) \times(0, \infty)$, it is easily seen that

$$
\alpha_{m}(k) \leqq \alpha_{n}(k), \quad m \leqq n \text { and } k \in \Lambda_{m} .
$$

We next observe that if $C^{2} \equiv \sup _{k \in Z^{d}} \sup _{\eta \in E} c_{k}(\eta)$, then

$$
\beta_{n}(k) \leqq e C\left(\alpha_{n}(k)\right)^{1 / 2}, \quad n \geqq 1 \quad \text { and } \quad k \in \Lambda_{n} .
$$

To see this, first note that there is nothing to prove if $\alpha_{n}(k) \geqq C^{2}$, since $\beta_{n}(k)$ is always less than or equal to $2 C^{2}$. If $\alpha_{n}(k) \leqq C^{2}$, let $0<\varepsilon \leqq 2$ be given and segregate the terms in the sum defining $\beta_{n}(k)$ into those for which $\left|\log \frac{\Gamma_{n}(k, A)}{\Gamma_{n}\left(k, A_{k}\right)}\right|<\varepsilon$ and those for which $\left|\log \frac{\Gamma_{n}(k, A)}{\Gamma_{n}\left(k, A_{k}\right)}\right| \geqq \varepsilon$. One then sees that:

$$
\begin{aligned}
\beta_{n}(k) & \leqq\left(e^{\varepsilon}-1\right) \sum_{A \subseteq A_{n}} \Gamma_{n}(k, A)+\frac{1}{\varepsilon} \alpha_{n}(k) \\
& \leqq \varepsilon e^{2} C^{2}+\frac{1}{\varepsilon} \alpha_{n}(k)
\end{aligned}
$$


In particular, we can take

$$
\varepsilon^{2}=\frac{\alpha_{n}(k)}{e^{2} C^{2}} \leqq 1 / e^{2}
$$

and thereby arrive at (1.20).

Now define

$$
\gamma_{1}=\sum_{k \in \Lambda_{1}} \alpha_{1}(k)
$$

and

$$
\gamma_{n}=\sum_{k \in \partial \Lambda_{n}} \alpha_{n}(k), \quad n \geqq 2 .
$$

Successively using (1.20), (1.18), (1.21), and Schwartz's inequality, we see that

$$
\sum_{1}^{N} \gamma_{n} \leqq e C K L^{(d-1) / 2} N^{(d-1) / 2} \gamma_{N}^{1 / 2}, \quad N \geqq 2
$$

Lemma 1.23. Let $\left\{\delta_{n}\right\}_{n=0}^{\infty}$ be a sequence of non-negative numbers with $\sum_{n=0}^{\infty} \delta_{n} \equiv\|\delta\|<\infty$. Let $\{h\}_{n=1}^{\infty}$ be a sequence of non-negative numbers with the property that

$$
f(N)=\sum_{n=1}^{N} h_{n} \leqq \sum_{n=1}^{N} \delta_{N-n} h_{n}^{1 / 2} u_{n}^{1 / 2}, \quad N \geqq 2,
$$

where $u_{n}>0$ and $u_{n+1} \geqq u_{n}$ for all $n$. If $\sum_{n=1}^{\infty} \frac{1}{u_{n}}=\infty$, then $h_{n}=0$ for all $n \geqq 1$.

Proof.

$$
\begin{aligned}
f^{2}(N) & \leqq\left(\sum_{n=1}^{N} \delta_{N-n}^{1 / 2} \delta_{N-n}^{1 / 2} h_{n}^{1 / 2} u_{n}^{1 / 2}\right)^{2} \\
& \leqq\|\delta\| \sum_{n=1}^{N} \delta_{N-n} h_{n} u_{n} \leqq\|\delta\| u_{N} \sum_{n=1}^{N} \delta_{N-n} h_{n} .
\end{aligned}
$$

Suppose $h_{n}>0$ for some $n$. Let $n_{0}$ be the smallest such $n$. Then $f^{2}(N) \geqq h_{n_{0}}^{2}$ for all $N \geqq n_{0}$. Thus for $N>n_{0}$

$$
\begin{aligned}
\frac{1}{u_{N}} & \leqq\|\delta\|\left[\sum_{n=n_{0}+1}^{N} \delta_{N-n} \frac{h_{n}}{f^{2}(N)}+\delta_{N-n_{0}} \frac{1}{h_{n_{0}}}\right] \\
& \leqq\|\delta\|\left[\sum_{n=n_{0}+1}^{N} \delta_{N-n}\left(\frac{1}{f(n-1)}-\frac{1}{f(n)}\right)+\delta_{N-n_{0}} \frac{1}{h_{n_{0}}}\right] .
\end{aligned}
$$

Thus

$$
\sum_{N=n_{0}+1}^{\infty} \frac{1}{\mathrm{u}_{N}} \leqq\|\delta\|\left[\|\delta\| \frac{2}{f\left(n_{0}\right)}\right]<\infty,
$$

which is a contradiction. 
Now set $\delta_{0}=e C K L^{(d-1) / 2}, \delta_{k}=0$ for $k \geqq 1$, and $u_{N}=N^{d-1}$. Applying Lemma 1.23 to (1.22) when $d=1$ or 2 , we now have that $\gamma_{n}=0$ for all $n \geqq 1$. But this means that $\alpha_{n}(k)=0$ for all $k \in \partial \Lambda_{n}$; and therefore, by $(1.21) \beta_{n}(k)=0$ for all $k \in \partial \Lambda_{n}$. Using this in (1.18), one arrives at the conclusion that

$$
\Gamma_{n}(k, A)=\Gamma_{n}\left(k, A_{k}\right), \quad n \geqq 2, \quad k \in \Lambda_{n} \quad \text { and } \quad A \leqq \Lambda_{n} .
$$

Since (1.24) certainly implies (1.8), the proof of Theorem 1.7 is now complete.

(1.25) Remark. Lemma 1.23 is more general than necessary to prove Theorem 1.7. We have included this more general result because it allows one to generalize Theorem 1.7 to include some situations which are not finite range by making the obvious modifications in the above proof. We have not bothered to include all the details of this generalization because this is not the direction in which it would be most interesting to generalize Theorem 1.7. What one really wants is to know whether Theorem 1.7 holds when $d \geqq 3$.

\section{Discussion}

If the potential, $\left\{J_{F}: F \in \hat{E}\right\}$, is such that there is only one Gibbs state, $\mu$, then under the hypotheses of Theorem 1.7

$$
\lim _{S \rightarrow \infty} \frac{1}{S} \int_{0}^{S} T_{t} f(\eta) d t=\int f d \mu
$$

for all $\eta \in E$ and $f \in C(E)$. It seems likely in this case that the stronger result $T_{t} f(\eta) \rightarrow \int f d \mu$ is also true; however, this has only been proved in special cases (see [3] and [5]). It is known, however, that even if $T_{t} f(\eta) \rightarrow \int f d \mu$ for all $\eta \in E$ and all $f \in C(E)$ the convergence may be extremely slow (see Remark 2.21 in [6]).

If there are many Gibbs states for $\left\{J_{F}: F \in \hat{E}\right\}$, then for some $\eta \in E$ and $f \in C(E)$, $T_{t} f(\eta)$ may not converge at all (see Section 3 of [3]). However if $\alpha$ is a limit point of $\frac{1}{S} \int_{0}^{S} T_{t} f(\eta) d t$ and if Theorem 1.7 applies, then we must have $\alpha=\mu(f)$ for some Gibbs state $\mu$ having potential $\left\{J_{F}: F \in \hat{E}\right\}$.

Theorem 1.7 is more interesting in two dimensions than it is in one dimension. In one dimension it is conceivable that for any uniformly positive, uniformly bounded $c_{k}$ 's satisfying $c_{k}(\eta)=c_{k}(\eta)$ if $|k-l|>L$ (but not necessarily associated with a potential) there is only one stationary measure for the semigroup. If this is true, then in one dimension Theorem 1.7 is a special case of a more general theorem; however in two dimensions this cannot be the case, since it is possible for the stochastic Ising model to have more than one stationary measure in two dimensions. Hence in two dimensions Theorem 1.7 cannot be a consequence of a theorem which guarantees uniqueness of the stationary measure in all finite range situations.

Aside from the details of the computation, we do not understand why this technique should fail in three or more dimensions and work in two dimensions. It is possible that the first derivative of the free energy does not contain enough information in three dimensions for the same reason that there may exist nonperiodic Gibbs states in three dimensions, even if the potential is translation 
invariant. More specifically, in one and two dimensions, it may be the case that if the $c_{k}$ 's are any strictly positive, translation invariant, continuous functions satisfying $c_{k}(\eta)=c_{k}(\eta)$ if $|k-l|>L$ (but not necessarily associated with a potential) and $v$ is any stationary measure for the semi-group, then $v$ is periodic. This is false in three dimensions as the stochastic Ising model shows; however, if it is true in one and two dimensions, it would immediately imply Theorem 1.7 (at least for translation invariant $\left(c_{k}\right.$ 's) since free energy arguments are very effective for periodic measures (see [7]).

Finally we would like to point out that the assumption that $c_{k}(\eta)>0$ for all $k$ and $\eta$ is critical. [This was used in showing that (1.8) is equivalent to $\mu$ being a Gibbs state and in the proof of Lemma 1.16.] For example, let $d=1$ and $J_{R}=1$ if $R=(k, k+1)$ for some $k$ and 0 otherwise. If $b_{k}$ in (1.2) is given by $b_{k}(\eta)=\left(1+\eta_{k-1}\right)$, then there are two stationary measures: the measure concentrated on $\eta_{k} \equiv-1$ and the Gibbs state for the potential $\left\{J_{R}\right\}$.

\section{References}

1. Higuchi, Y., Shiga,T.: Some results on Markov processes in infinite lattice spin systems. J. Math. Kyoto Univ. 15, 211-229 (1975)

2. Holley, R. : Free energy in a Markovian model of a lattice spin system. Commun. math. Phys. 23, 8799 (1971)

3. Holley, R.: Recent results on the stochastic Ising model. Rocky Mountain J. Math. 4, 479-496(1974)

4. Holley, R., Stroock, D. : A martingale approach to infinite systems of interacting process. Ann. Prob. 4, 195-228 (1976)

5. Holley, R., Stroock,D.: Applications of the stochastic Ising model to the Gibbs states. Commun. math. Phys. 48, 249-265 (1976)

6. Holley, R., Stroock, D. : $L_{2}$ theory for the stochastic Isimg model. Z. Wahrscheinlichkeitstheorie verw. Geb. 35, 87-101 (1976)

7. Moulin Ollagnier,J., Pinchon,D.: Free energy in spin-flip processes is non-increasing. Commun. math. Phys. 55, 29-35 (1977)

Communicated by J. L. Lebowitz

Received November 18, 1976 
